\title{
A Second-Look at the Repeat Resections of Bladder Tumors
}

\author{
Madison Lyon ${ }^{\mathrm{a}, \mathrm{c}}$, Sharon White ${ }^{\mathrm{a}}$, Taylor Goodstein ${ }^{\mathrm{a}}$, Madeline Koerner ${ }^{\mathrm{b}}$, Alexandra Colvin ${ }^{\mathrm{a}}$, \\ Colin I. O’Donnell ${ }^{\mathrm{a}}$, Janine Olivera, Shandra Wilson ${ }^{\mathrm{a}}$
}

\begin{abstract}
Background: Research looking at the effects of repeat transurethral resection of bladder tumor (TURBT) on long-term survival and recurrence of bladder cancer has mixed results, with some studies showing improvement and others showing unclear benefit. The purpose of this current study is to evaluate the differences in recurrence rates, progression, and survival for patients with non-muscle invasive bladder cancer (NMIBC) who underwent a second TURBT 2 - 6 weeks after an initial TURBT, as compared to patients who only had an initial TURBT.
\end{abstract}

Methods: We performed a retrospective analysis of patients who received a TURBT at our institution over a 12-year period (2005 2017). Patients 18 years or older with high-grade pT1 or pTis pathology on initial TURBT were included. Patients with low-grade, pTa, or stage pT2 or greater on initial TURBT and patients with variant histology other than urothelial carcinoma were excluded from the study. Differences in overall survival, recurrence rates, and cancer progression were analyzed using Cox regression to event and Poisson regression to number of occurrences in patients who had repeat TURBT vs. those who did not. Cancer progression was measured as time to cystectomy.

Results: One hundred fifty-three patients with a diagnosis of highgrade NMIBC were included in the study. Forty-six patients $(30.1 \%)$ had a repeat TURBT. There was no significant difference in baseline characteristics including age, stage, or gender between the two groups. After a median follow-up of 67.5 months (range $7.5-200$ months), there was no significant difference in overall survival (OS) $(\mathrm{P}=0.63)$, cancer progression $(\mathrm{P}=0.51)$ or recurrence rates $(\mathrm{P}=$ 0.60 ) for patients who underwent second-look TURBT compared to those who did not. Of those patients who underwent repeat TURBT, $32(69.6 \%)$ had residual tumor and $13(28.2 \%)$ had a change in stage. Of the patients that had a change in stage, five were upstaged to muscle invasive (pT2) disease.

Manuscript submitted September 6, 2018, accepted September 24, 2018

aDivision of Urology, Department of Surgery, University of Colorado School of Medicine, University of Colorado Hospital (UCH), 12605 E. 16th Ave, Aurora, CO 80045, USA

bepartment of Urology, Louisiana State University, 1901 Perdido Street, New Orleans, LA 70112, USA

${ }^{\mathrm{c} C}$ Corresponding Author: Madison Lyon, Division of Urology, Department of Surgery, University of Colorado School of Medicine, University of Colorado Hospital (UCH), 12605 E. 16th Ave, Aurora, CO 80045, USA.

Email: madison.lyon@ucdenver.edu

doi: https://doi.org/10.14740/wjnu361w
Conclusions: Our analysis did not show an association between second-look TURBT with overall survival, progression, or recurrence rate of bladder cancer as compared to no second-look TURBT. However, there was a small rate of upstaging to pT2 disease on repeat TURBT. Even though there was no change in OS, repeat TURBT has an important role in finding T2 disease, and this is so critical that continuing with this conservative approach of a second TURBT is still recommended.

Keywords: Bladder cancer; TURBT; Transurethral resection; Bladder; Tumor; Bladder tumor; Urologic oncology

\section{Introduction}

Non-muscle invasive bladder cancer (NMIBC) comprises the vast majority of bladder cancer diagnoses, and transurethral resection of bladder tumor (TURBT) is the basis of diagnosis and treatment [1]. The 2016 guidelines from the American Urological Association and Society of Urologic Oncology recommend repeat TURBT for patients that had an incomplete resection of a NMIBC or had evidence of T1 disease, and recommend that repeat TURBT be considered in patients with high risk, high grade Ta disease [2-4].

These guidelines are supported by multiple studies showing that initial TURBT misses residual tumor at a rate of 27$76 \%$ and does not detect muscle invasive disease at a rate of $5-38 \%$, which translates to a significant risk for progression and understaging of disease on initial evaluation [1, 5-7]. There have also been studies that show that repeat TURBT is associated with increased response to intravesical BCG and mitomycin $[6,8,9]$. However, the clinical significance of these findings remains unclear. Results from studies on long-term survival and recurrence are mixed, with some showing improvement with repeat TURBT $[6,8-10]$, and others showing unclear benefit and stressing the need for further study $[7,11]$.

The current study is a retrospective analysis examining the differences in recurrence rates, progression, and survival for patients with NMIBC who underwent second TURBT 2 - 6 weeks after an initial TURBT, compared to patients who only had an initial TURBT.

\section{Materials and Methods}

We performed a retrospective analysis of patients who received 
Table 1. Patient Characteristics

\begin{tabular}{llll}
\hline & Repeat TURBT & No repeat TURBT & Pvalue \\
\hline $\begin{array}{l}\text { Initial TURBT pathology } \\
\text { pTis }\end{array}$ & $1(2 \%)$ & $9(8 \%)$ & 0.15 \\
pT1 & $45(98 \%)$ & $98(92 \%)$ & 0.15 \\
Patient gender & & & \\
Male & $39(85 \%)$ & $83(78 \%)$ & 0.31 \\
Female & $7(15 \%)$ & $24(22 \%)$ & 0.31 \\
BCG received & $30(65 \%)$ & $65(60 \%)$ & 0.73 \\
\hline
\end{tabular}

a TURBT of a bladder tumor at the University of Colorado Hospital over a 12-year period, January 2005 through December 2017. Patients were identified using ICD-9 diagnostic codes for "Cystourethroscopy with biopsy," "Cystourethroscopy with fulguration and/or resect large bladder tumor/s," "Cystourethroscopy w/fulguration trigone/bladder neck/prostatic fossa/urethra, etc.," or "Cystourethroscopy, with insertion of indwelling ureteral stent." Patients 18 years or older with high-grade pT1 or pTis pathology on initial TURBT were included. Patients with low-grade, pTa, or stage pT2 or greater on initial TURBT and patients with variant histology other than urothelial carcinoma were excluded from the study. A total of four different surgeons performed the procedures. The electronic medical record was reviewed to collect patient demographic and clinical data. Survival data were collected by review of the electronic medical record and by online obituary search.

The primary objective of this study was to examine the rates of overall survival (OS) in patients who underwent repeat TURBT compared to those who did not undergo repeat TURBT, among patients with high-grade pT1 or pTis pathology on initial TURBT. A repeat TURBT was defined as a TURBT of the same area within 2 - 6 weeks after an initial TURBT. Secondary endpoints examined included rates of upstaging on second TURBT, cancer progression, and time to recurrence. Cancer progression was measured as time to cystectomy. A recurrence was defined as another bladder tumor identified on later TURBT performed more than 6 weeks after resection of the initial tumor. Data on BCG and mitomycin administration were also collected and analyzed for impact on the outcomes of interest. Differences in OS, recurrence rates, and cancer progression were analyzed using Cox regression to event and Poisson regression to number of occurrences in patients who had repeat TURBT vs. those who did not. Statistical analysis was performed using SAS (C) 9.4 TS Level $1 \mathrm{M} 3$, and R version 3.4.2 (C) The R Foundation for Statistical Computing.

\section{Results}

A total of 154 patients were identified. One patient who died, but did not have a known date of death, was additionally excluded from the study. The final analytic dataset included 153 patients with a diagnosis of high-grade pT1 or pTis non- muscle invasive urothelial carcinoma. Ten patients were initially staged pTis $(6.5 \%)$, and 143 patients were staged as pT1 (93.5\%). The cohort was comprised of 31 women $(20.3 \%)$ and 122 men $(79.7 \%)$. Forty-six (30.1\%) patients had a repeat TURBT. There was no significant difference in baseline characteristics including age $(\mathrm{P}=0.61)$, gender $(\mathrm{P}=0.61)$, or stage $(\mathrm{P}=0.28)$ between the two groups. There was no significant difference in the proportion of patients who received BCG in each group $(\mathrm{P}=0.73$; Table 1$)$.

After a median follow-up of 67.5 months (range: 7.5 - 200 months), there was no significant difference in $\mathrm{OS}(\mathrm{P}=0.63)$ (Fig. 1), cancer progression $(\mathrm{P}=0.51)$ or recurrence rates $(\mathrm{P}=$ $0.60)$ for patients who underwent second-look TURBT compared to those who did not (Table 2). There was also no significant difference in time to death $(\mathrm{P}=0.58)$, time to cystectomy $(\mathrm{P}=0.21)$, and time to recurrence $(\mathrm{P}=0.82)$. Of those patients who underwent repeat TURBT, $32(69.6 \%)$ had residual tumor, of which $13(28.2 \%)$ had a change in stage. Of the patients that had a change in stage, five were upstaged to muscle invasive (pT2) disease, two patients who had pT1 disease on their initial resection had $\mathrm{pTa}$ on repeat TURBT, and six patients who initially had pT1 disease had pTis on repeat TURBT.

\section{Discussion}

In this cohort of patients with a diagnosis of high-grade pT1 or pTis urothelial carcinoma on initial TURBT, we observed no significant difference in OS, cancer progression, or recurrence rates for patients who underwent second-look TURBT when compared to those who did not. According to the current guidelines, repeat TURBT is recommended for patients who had an incomplete resection of NMIBC or had evidence of T1 disease, and it should be considered in patients with high risk, high grade Ta disease [2]. This is supported by several studies which have shown that repeat TURBT often reveals residual tumor and/or results in upstaging, however the data surrounding the survival and recurrence benefits of repeat TURBT are mixed $[2,6,8,12,13]$.

In a prospective cohort study, Ali et al followed 91 patients with T1 and Ta on initial TURBT [14]. After second TURBT, 22 patients had an increased stage, leading to modification in treatment. This led the authors to conclude that second-look TURBT should be done for accurate staging of bladder cancer [12]. In our study, of the patients that underwent second- 


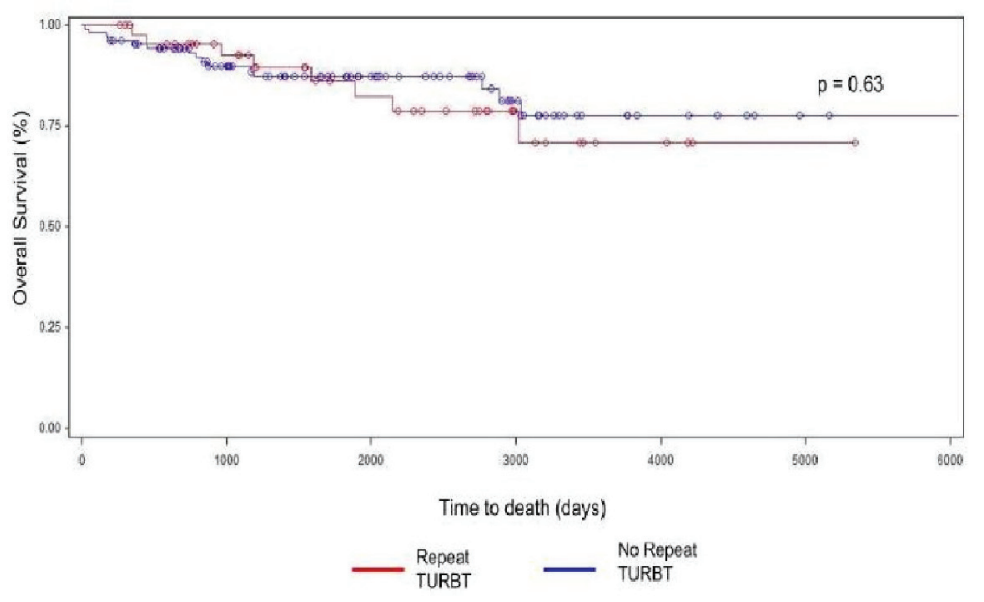

Figure 1. Overall survival for patients who underwent repeat TURBT vs. those that did not.

look TURBT, $10.9 \%$ were upstaged to muscle-invasive disease after repeat TURBT. While this is a small fraction of the overall cohort, upstaging to pT2 disease, of course, has significant clinical implications. One study suggests that muscularis propria is missing in approximately $51 \%$ of TURBT specimens [13]. Lack of muscularis propria with the specimen on initial TURBT is not a part of the guidelines for pursuing repeat TURBT, even though it represents a less than ideal tissue biopsy. The overall rate of muscularis present in the study population can give a sense of the quality of resection being performed. However, we did not analyze pathology reports to determine which TURBT specimens included muscularis propria, and missing muscularis propria on initial TURBT could be a reason for later upstaging during repeat TURBT.

The recommendation for repeat TURBT is also supported by multiple studies demonstrating high rates of residual tumor on repeat TURBT, ranging from $27-76 \%[2,6,8,12,13]$. The findings from our study were in line with the current literature, showing a rate of residual tumor of $69.6 \%$ on repeat TURBT. The high rate of residual tumor after initial TURBT is concerning, and is one of the main reasons, along with accurate staging, which led to adopting repeat TURBT into urologists' practice $[1,5]$. Our study, however, shows that removing residual tumor on repeat TURBT did not end up improving outcomes for those patients.

In addition to potential the implications of repeat TURBT on accurate staging and removal of residual tumor, in previous studies, repeat TURBT has been shown to reduce tumor recurrence and progression. Angulo et al found that a second TURBT reduced recurrence at 3 months and progression at 1 year, but did not significantly affect long-term recurrence, progression, or cancer death [11]. It is notable to mention that an analysis of several randomized controlled trials showed variable rates of bladder cancer recurrence between different institutions, suggesting variability between providers [13, 15]. Interestingly, Hashine et al also found that second TURBT was not associated with a reduction in intravesical recurrence regardless of intravesical BCG, but second TURBT did improve overall survival in their study [7].

In our cohort, slightly less than one-third of patients underwent repeat TURBT. At our institution the practice of a second-look TURBT was adopted in 2010, which would account for the comparatively lower rate of repeat TURBT performed in our population. This study was limited by our reliance on retrospective chart review for data collection. Furthermore, because we collected data from a single tertiary care center, many of the patients included in the study returned to their primary urologists for follow-up, which limited our length of follow-up and may have resulted in a selection bias. An additional variable analyzed by other studies is the interaction of repeat TURBT with immunotherapies such as BCG and mitomycin. Multiple studies have suggested that repeat TURBT was associated with an improved response to immunotherapy; however, this is a future area of study not included in our present analysis $[6,8]$.

In conclusion, this study found no difference in OS, cancer progression, or recurrence in patients undergoing repeat TURBT as compared to those who did not. Our findings support prior data showing a small rate of upstaging to pT2 disease on repeat TURBT. Even though there was no change in OS, repeat TURBT has an important role in finding T2 disease, and this is so critical, that continuing with this conservative approach of a second TURBT is still recommended. Further prospective studies are needed to examine the impact of repeat

Table 2. Outcomes of Patients Who Had a Repeat TURBT vs. Those That Did Not

\begin{tabular}{llll}
\hline & Repeat TURBT & No repeat TURBT & P value \\
\hline Overall survival (\% alive) & $38(83 \%)$ & $92(86 \%)$ & 0.63 \\
Progression to cystectomy & $11(24 \%)$ & $20(19 \%)$ & 0.51 \\
Tumor recurrence & $20(43 \%)$ & $52(49 \%)$ & 0.60 \\
\hline
\end{tabular}


TURBT on outcomes of NMIBC.

\section{Funding}

None.

\section{Conflict of Interest}

The authors have no conflict of interest to disclose.

\section{References}

1. Hall MC, Chang SS, Dalbagni G, Pruthi RS, Seigne JD, Skinner EC, Wolf JS, Jr., et al. Guideline for the management of nonmuscle invasive bladder cancer (stages Ta, T1, and Tis): 2007 update. J Urol. 2007;178(6):23142330.

2. Chang SS, Boorjian SA, Chou R, Clark PE, Daneshmand S, Konety BR, Pruthi R, et al. Diagnosis and treatment of non-muscle invasive bladder cancer: AUA/SUO Guideline. J Urol. 2016;196(4):1021-1029.

3. Babjuk M, Bohle A, Burger M, Capoun O, Cohen D, Comperat EM, Hernandez V, et al. EAU Guidelines on non-muscle-invasive urothelial carcinoma of the bladder: update 2016. Eur Urol. 2017;71(3):447-461.

4. American Urological Association. Treatment of nonmetastatic muscle-invasive bladder cancer: AUA/ASCO/ ASTRO/SUO Guideline. Available at: http://www. auanet.org/guidelines/muscle-invasive-bladder-cancernew-(2017). (Accessed: 19th December 2017).

5. Herr HW. The value of a second transurethral resection in evaluating patients with bladder tumors. J Urol. 1999;162(1):74-76.

6. Herr HW. Restaging transurethral resection of high risk superficial bladder cancer improves the initial response to bacillus Calmette-Guerin therapy. J Urol. 2005;174(6):2134-2137.

7. Hashine K, Ide T, Nakashima T, Hosokawa T, Ninomiya I,
Teramoto N. Results of second transurethral resection for high-grade T1 bladder cancer. Urol Ann. 2016;8(1):10-15.

8. Sfakianos JP, Kim PH, Hakimi AA, Herr HW. The effect of restaging transurethral resection on recurrence and progression rates in patients with nonmuscle invasive bladder cancer treated with intravesical bacillus Calmette-Guerin. J Urol. 2014;191(2):341-345.

9. Divrik RT, Yildirim U, Zorlu F, Ozen H. The effect of repeat transurethral resection on recurrence and progression rates in patients with $\mathrm{T} 1$ tumors of the bladder who received intravesical mitomycin: a prospective, randomized clinical trial. J Urol. 2006;175(5):1641-1644.

10. Divrik RT, Sahin AF, Yildirim U, Altok M, Zorlu F. Impact of routine second transurethral resection on the long-term outcome of patients with newly diagnosed pT1 urothelial carcinoma with respect to recurrence, progression rate, and disease-specific survival: a prospective randomised clinical trial. Eur Urol. 2010;58(2):185-190.

11. Angulo JC, Palou J, Garcia-Tello A, de Fata FR, Rodriguez O, Villavicencio $\mathrm{H}$. Second transurethral resection and prognosis of high-grade non-muscle invasive bladder cancer in patients not receiving bacillus Calmette-Guerin. Actas Urol Esp. 2014;38(3):164-171.

12. Lipsker A, Hammoudi Y, Parier B, Drai J, Bahi R, Bessede T, Patard JJ, et al. [Should we propose a systematic second transurethral resection of the bladder for all highrisk non-muscle invasive bladder cancers?]. Prog Urol. 2014;24(10):640-645.

13. Herr H, Dalbagni G. Is 'second-look' (re-staging) transurethral resection of bladder tumours a new standard of care? Arab J Urol. 2011;9(1):7-10.

14. Ali MH, Ismail IY, Eltobgy A, Gobeish A. Evaluation of second-look transurethral resection in restaging of patients with nonmuscle-invasive bladder cancer. J Endourol. 2010;24(12):2047-2050.

15. Brausi M, Collette L, Kurth K, van der Meijden AP, Oosterlinck W, Witjes JA, Newling D, et al. Variability in the recurrence rate at first follow-up cystoscopy after TUR in stage Ta T1 transitional cell carcinoma of the bladder: a combined analysis of seven EORTC studies. Eur Urol. 2002;41(5):523-531. 